\title{
Accurate miscut angle determination for spherically bent Bragg crystals
}

D. S. Covita, M. Ay, S. Schlesser, D. Gotta, L. M. Simons, E.-O. Le Bigot, and J. M. F. dos Santos

Citation: Review of Scientific Instruments 79, 033102 (2008);

View online: https://doi.org/10.1063/1.2884149

View Table of Contents: http://aip.scitation.org/toc/rsi/79/3

Published by the American Institute of Physics

\section{Articles you may be interested in}

Measurement of the miscut angle of crystal surfaces vicinal to major crystal planes by x-ray diffractometry at glancing incidence

Applied Physics Letters 53, 2042 (1998); 10.1063/1.100313

Accurate monocrystal miscut angle determination by $\mathrm{x}$-ray diffraction on a wedge

Applied Physics Letters 63, 458 (1998); 10.1063/1.110023

Precise orientation of single crystals by a simple x-ray diffraction rocking curve method

Review of Scientific Instruments 76, 036106 (2005); 10.1063/1.1867392

A seven-crystal Johann-type hard x-ray spectrometer at the Stanford Synchrotron Radiation Lightsource Review of Scientific Instruments 84, 053102 (2013); 10.1063/1.4803669

Calculation of the Johann error for spherically bent $\mathrm{x}$-ray imaging crystal spectrometers

Review of Scientific Instruments 81, 10 E329 (2010); 10.1063/1.3491195

Characterization of a charge-coupled device array for Bragg spectroscopy

Review of Scientific Instruments 77, 043107 (2006); 10.1063/1.2194485

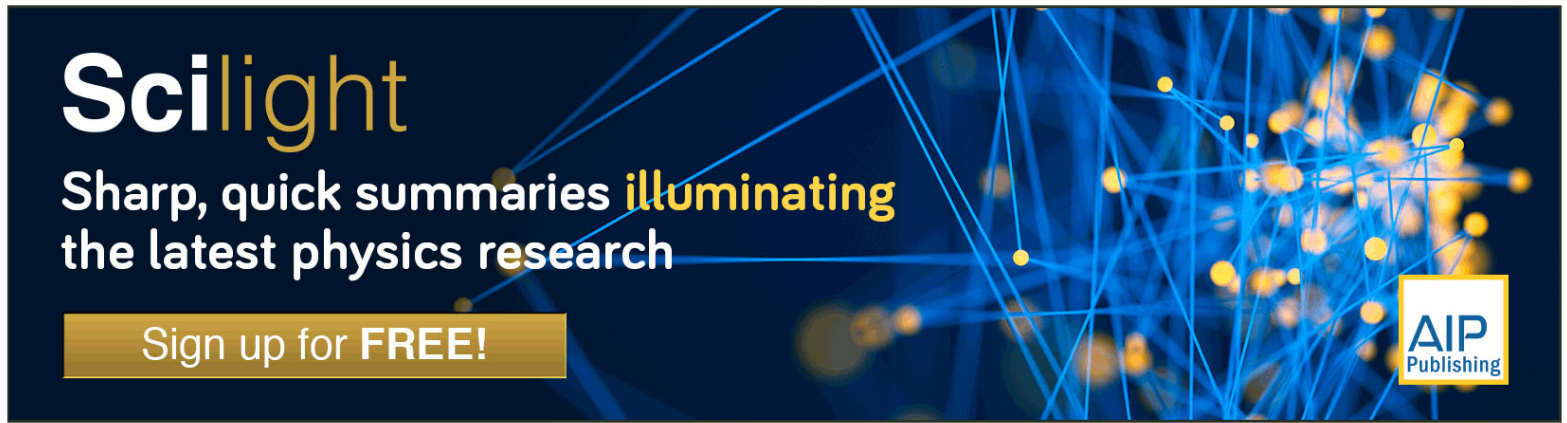




\title{
Accurate miscut angle determination for spherically bent Bragg crystals
}

\author{
D. S. Covita, ${ }^{1, a)}$ M. Ay, ${ }^{2}$ S. Schlesser, ${ }^{4}$ D. Gotta,${ }^{3}$ L. M. Simons,,${ }^{5, b)}$ E.-O. Le Bigot, ${ }^{4}$ and \\ J. M. F. dos Santos ${ }^{1}$ \\ ${ }^{1}$ Departamento de Física, Universidade de Coimbra, 3004-516 Coimbra, Portugal \\ ${ }^{2}$ Laboratory for Neutron Scattering, ETHZ and Paul Scherrer Institut, CH-5232 Villigen PSI, Switzerland \\ ${ }^{3}$ Institut für Kernphysik, Forschungszentrum Jülich, D-52425 Jülich, Germany \\ ${ }_{5}^{4}$ Laboratoire Kastler Brossel, UPMC-Paris 6, ENS, CNRS; Case 74, 4 place Jussieu, 75005 Paris, France \\ ${ }^{5}$ Laboratory for Particle Physics (LTP), Paul Scherrer Institut, CH-5232 Villigen PSI, Switzerland
}

(Received 11 January 2008; accepted 30 January 2008; published online 5 March 2008)

\begin{abstract}
Spherically bent crystals are used as analyzers in high-resolution spectroscopy, in particular, in low count-rate applications such as exotic-atom research. The focal conditions are determined not only by the bending radius and the Bragg angle but also by the crystal cut angle between its surface and the reflecting crystal planes, along with their orientation with respect to the direction of dispersion. We describe a simple but precise method for measuring the cut angle and its orientation for mounted spherically bent crystals, by combining x-ray diffraction and laser optical alignment, which can be easily performed with standard x-ray laboratory equipment. () 2008 American Institute of Physics.

[DOI: 10.1063/1.2884149]
\end{abstract}

\section{INTRODUCTION}

Large area spherically bent Bragg crystals are used in the $\mathrm{x}$-ray spectroscopy of exotic atoms in the few $\mathrm{keV}$ range. ${ }^{1-3}$ Owing to their focusing properties, they reduce measuring times by an order of magnitude compared to plane crystal arrangements. In this (Johann-type) setup, ${ }^{4}$ defocusing due to the imaging properties can be well controlled by choosing appropriate dimensions for x-ray source, crystal, and its radius of curvature, in combination with a large area positionsensitive detector. 5

A general problem is that the precise knowledge of the orientation of the lattice planes of the slabs, cut from a crystal bulk and subsequently polished, may be lost. Deviations are due not only to an imperfect determination of the orientation of the bulk material but especially from alignment problems of the sawing machine. Spherically bent crystals are made by forcing thin slabs into a spherical shape; therefore the crystal cut angle (miscut) can be assumed to be constant over the whole crystal surface. In our case, the plates follow the curvature of a high-quality polished spherical glass lens kept in place by molecular forces (Fig. 1). The meridional orientation may also change during the contacting process. In fact, x-ray diffraction diagnostics are usually not available during the industrial fabrication procedure which is necessary for achieving highest polishing quality. Hence, after mounting the crystal, determining the miscut and its orientation is essential.

A miscut is characterized by an angle $\alpha$ between the crystal surface and the reflecting planes. As is seen from Guinier's focusing condition for cylindrically bent crystals, ${ }^{6}$

\footnotetext{
${ }^{\text {a) }}$ Author to whom correspondence should be addressed. Electronic mail: daniel.covita@psi.ch. Also at LTP, PSI, CH-5232 Villigen, Switzerland.

${ }^{b)}$ Present address: Departamento de Física da Universidade de Coimbra, 3004-516 Coimbra, Portugal.
}

the crystal cut angle contributes to the focal positions, i.e., in the distances between crystal (C), detector (D), and source (S) (Fig. 2),

$$
\begin{aligned}
& \overline{\mathrm{CD}}=R_{c} \sin \left(\Theta_{B}+\alpha\right), \\
& \overline{\mathrm{SC}}=R_{c} \sin \left(\Theta_{B}-\alpha\right) .
\end{aligned}
$$

For $\alpha=0$, the Guinier condition reduces to the usual (meridional) focusing condition for the symmetric Bragg case, $\overline{\mathrm{CD}}=R_{c} \sin \Theta_{B}$, where $\Theta_{B}$ is the Bragg angle according to Bragg's law and $R_{c}$ the crystal's bending radius. Similarly, the knowledge of $\alpha$ is equally important when using asymmetric Bragg reflections. For the sign of $\alpha$, we follow Guinier's convention, where a positive angle corresponds to a larger crystal-detector distance.

Equations (1) and (2) are only valid for a particular orientation of the crystallographic planes, i.e., when the directions of dispersion and the meridional plane are perpendicular; it also neglects bending itself. ${ }^{7}$ An arbitrary orientation of the miscut plane is equivalent to a rotation of the meridional plane by an angle $\varphi$ around the diameter $R_{c}$ of Fig. 2. The effective miscut $\alpha^{\prime}$ can then be obtained as the projection $\alpha \cos \varphi$. Rewriting Eq. (1) as $\overline{\mathrm{CD}}=R_{c} \sin \Theta_{B} \cos \alpha^{\prime}$ $+R_{c} \cos \Theta_{B} \sin \alpha^{\prime}$ and assuming a small miscut $(\alpha \approx 0)$, one obtains for any orientation $\varphi$ the focusing condition

$$
\overline{\mathrm{CD}}=R_{C} \sin \Theta_{B}+R_{C} \cos \Theta_{B} \sin (\alpha \cos \varphi) .
$$

As expected, an orientation according to $\varphi=90^{\circ}$ or $270^{\circ}$ does not change the focal length: rays in the plane of Fig. 2 are simply reflected above or below the symmetry plane, which can be corrected by tilting the crystal but leads to a topbottom asymmetry of the reflection.

The following example reveals the importance of a precise determination of both the miscut and its orientation: for a $\mathrm{Si}(111)$ crystal and $\mathrm{x}$-rays of the $3 p-1 s$ transition in pionic hydrogen $(2.89 \mathrm{keV})$ the Bragg angle is $\Theta_{B}=43.2^{\circ} .^{3} \mathrm{~A}$ mis- 


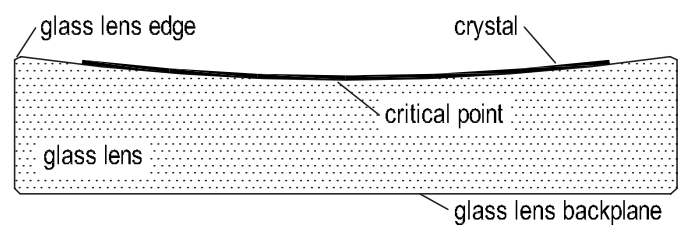

FIG. 1. Cross section of the crystal mounting on a concave glass lens of $3 \mathrm{~m}$ curvature, $12 \mathrm{~cm}$ diameter, and $3 \mathrm{~cm}$ thickness. The thickness of the crystal slabs is about $0.2-0.3 \mathrm{~mm}$ (curvature and crystal thickness not to scale).

cut as small as $0.1^{\circ}$ results in a change of the focal length of about $4 \mathrm{~mm}$ or $0.2 \%$ for $R_{c}=2.98 \mathrm{~m}$. If such a miscut is ignored and the detector is positioned at the supposed focal position, the displacement of the focus broadens the line by about $10 \mathrm{~s}$ of arc (for a horizontal crystal aperture of $60 \mathrm{~mm}$ ); this is to be compared to the intrinsic resolution of the silicon crystal of about $26 \mathrm{~s}$ of arc. ${ }^{8}$

\section{X-RAY DIFFRACTION BASED TECHNIQUES}

Techniques for determining the orientation of lattice planes within crystal blocks by diffraction methods are well established, ${ }^{9}$ in particular, for measuring the orientation of epitaxial layers and implantation profiles. ${ }^{10-14}$ The methods are based on angle differences and/or rocking curve width measurements, among others, with strongly asymmetric reflection setups. When adapted to flat specimen ${ }^{14}$ or a special form of the crystal material, ${ }^{13}$ they are not easily applicable to the spherically bent crystal setup.

The only alignment required for the method described in this paper is an adjustment of the x-ray spectrometer to the critical point (Fig. 1) where the bent surface is parallel to the reference plane defined by the spectrometer sample holder being perpendicular to the rotation axis (Fig. 3). The critical point is always the lowest point and, so, no further tilt adjustment is needed.

\section{CRYSTAL ALIGNMENT}

In practice, the lowest point does not coincide with the geometrical center of the crystal mounting. Therefore, at first the center offset of the critical point is found by means of a laser beam reflected from the crystal surface onto a screen. When rotating the crystal-lens mounting, the position of the

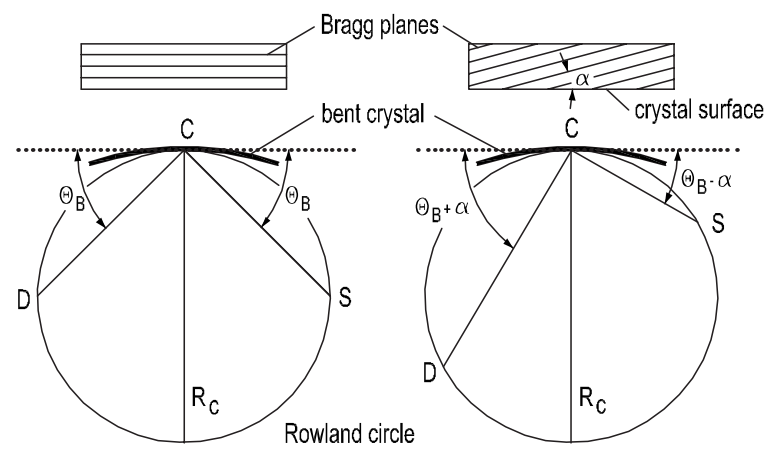

FIG. 2. Bragg reflection and focusing condition without and with miscut $\alpha$ for a bent crystal setup in the symmetry plane. The left drawing corresponds to the symmetric Bragg case, the right one to the asymmetric one for a miscut orientation $\varphi=0^{\circ}$ (adapted from Ref. 6).

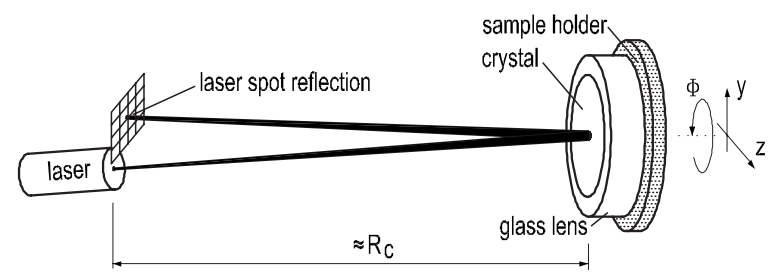

FIG. 3. Schematic of the method used for positioning the critical point (Fig. 1) onto the holder rotation axis.

reflected light remains unchanged if the critical point is on the axis of rotation, as is required by this method (Fig. 3). Otherwise, the reflected spot describes an ellipse. The closer the crystal sphere center to the rotation axis, the smaller the ellipse axes; the ellipse eventually collapses into a spot. During this procedure, the laser and the rotation axes are kept fixed and the sample holder, together with the crystal mounting clamped on it, is moved along the $y$ and $z$ coordinate in order to position the critical point onto the rotation axis (Fig. 3). The accuracy of this procedure depends on the surface to screen distance. In addition, the laser spot diameter can limit the accuracy. Placing the laser's aperture at a distance $R_{c}$ the crystal acts as a concave mirror, and the crystal will focus the reflected beam also at that distance.

In the absence of any miscut $(\alpha=0)$, an x-ray beam hitting the crystal surface at the critical point is always Bragg reflected under the angle $\Omega_{0}=\Theta_{B}$, whatever the holder rotation angle. For nonzero values of $\alpha$, the reflection angle $\Omega$ with respect to the reference plane varies periodically around $\Omega_{0}$ according to

$$
\Omega=\Omega_{0}+\alpha \cos \left(\Phi-\Phi_{0}\right),
$$

where $\varphi=\Phi-\Phi_{0}$ is the actual orientation. The angle $\Phi$ is measured relative to an arbitrary reference mark placed on the rim of the glass lens; the phase $\Phi_{0}$ represents the direction of the miscut with respect to the reference mark. Miscut angle and orientation $\left(\alpha, \Phi_{0}\right)$ are then obtained through a fit of the $\Omega(\Phi)$ curve (Fig. 4 ). During the x-ray sample spectrometer alignment, it was necessary to redefine the zero of the angle encoder. This explains the small offset on the $\Omega(\Phi)$ curves, which are not vertically centered at the corresponding Bragg angle $\Theta_{B}$. However, this has no impact on the extracted values of $\alpha$ and $\Phi_{0}$, as can be seen in Eq. (4).

\section{MEASUREMENT}

The measurements were performed with a SEIFERT® XRD 3003 PTS high-resolution x-ray diffraction spectrometer, which provides a collimated and monochromatic beam of $\mathrm{Cu} K \alpha$ x-rays $(8.041 \mathrm{keV})$. A sample spectrometer x-ray alignment was first performed. The optical alignment described above was done with a general-purpose gas laser $(660 \mathrm{~nm})$. The light spots were, respectively, about 6 and $0.5 \mathrm{~mm}$ on the crystal surface and on the screen catching the reflected laser beam. The x-ray beam was collimated to $1 \mathrm{~cm}$ in height and $1 \mathrm{~mm}$ in width, about $20 \mathrm{~cm}$ away from the sample. The collimator width at the detector side was $0.5 \mathrm{~mm}$ and its distance was also $20 \mathrm{~cm}$. 

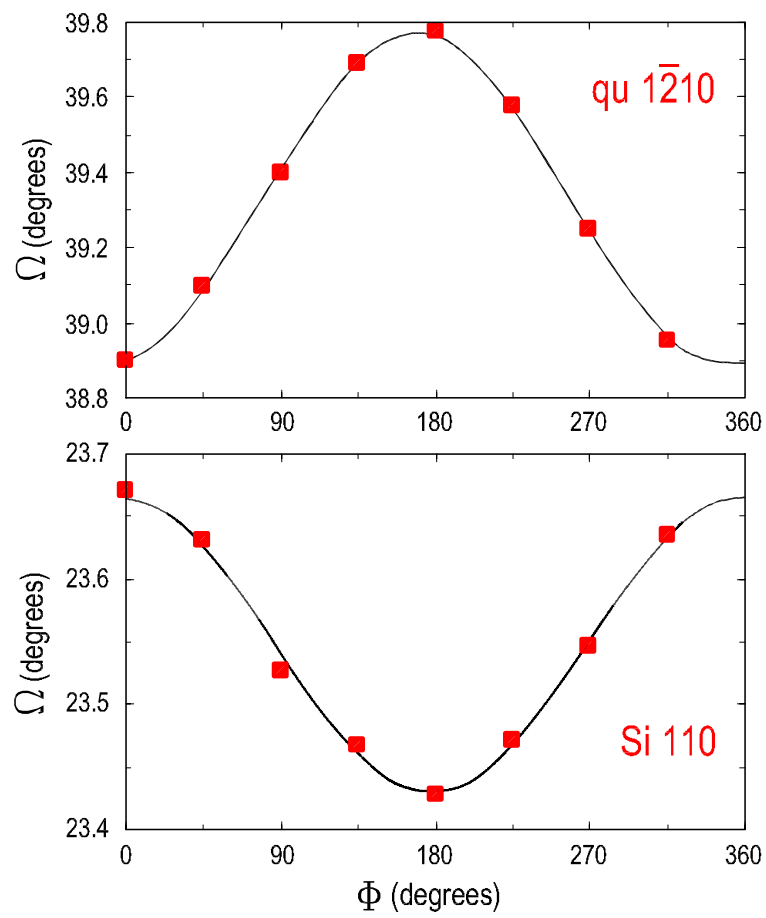

FIG. 4. (Color online) Variation of the reflection angle $\Omega$ for second order Bragg diffraction of the $\mathrm{Cu} K \alpha$ x-rays for a quartz (1 $\overline{2} 10)$ crystal [corresponding to $\Theta_{B}=39.41^{\circ}$ (Ref. 8) $]$ and a Si (110) crystal $\left[\Theta_{B}=23.67^{\circ}\right.$ (Ref. 8)]. The experimental errors for $\Omega$ are smaller than the dots depicting the data points. The solid line is a fit according to Eq. (4).

A set of 13 spherically bent crystals was characterized. As an example of a relatively large and of a relatively small miscut, the results for a quartz (12 10$)$ and a silicon (110) crystal are shown (Fig. 4). The fit to the predicted cosine behavior [Eq. (4)] is almost perfect. The angles $\alpha$ and $\Phi_{0}$ obtained for the quartz crystal are $0.442^{\circ} \pm 0.005^{\circ}$ and $169.0^{\circ} \pm 0.7^{\circ}$ and for silicon $0.119^{\circ} \pm 0.004^{\circ}$ and $-3.0^{\circ} \pm 2.0^{\circ}$, respectively.

\section{DISCUSSION}

In addition to the fit error, experimental uncertainties must be considered. The hitting position of the collimated $\mathrm{x}$-ray beam may deviate from the critical point by the step width of the $y-z$ movement, which was about $0.5 \mathrm{~mm}$. This results in ellipses with an axis up to $4 \mathrm{~mm}$ corresponding to an uncertainty of $0.019^{\circ}$ on the cut angle for crystals with $3 \mathrm{~m}$ bending radius. As the laser spot is less than $1 \mathrm{~mm}$, the step width dominates the error of $\alpha$ in this setup. As mentioned, the orientation angle $\Phi_{0}$ of the miscut is measured relative to a reference mark on the glass lens. The accuracy $\Delta \Phi_{0}$ for the mounting of the glass lens on our sample holder is better than $2^{\circ}$.

Of great advantage is the fact that the results are obtained by fitting a simple cosine function, which makes a specific adjustment of the meridional plane unnecessary. Obviously, the accuracy of the method scales linearly with the dimensions of the laser setup. The accuracy can easily be increased by adding data points to such curves-being advantageous for very small cut angles-as far as mechanical limitations appear. Furthermore, almost no restrictions for the Bragg angle arise because any diffraction angle accessible by the diffraction spectrometer can be used. Noteworthy is that the method can also be applied to the measurement of the miscut at any point of the crystal surface, by using a holder capable of tilting the crystal mounting.

\section{SUMMARY}

We describe a simple and precise method for determining or checking the miscut and its orientation of spherically bent crystal mountings, which have been used in ultimate resolution x-ray spectroscopy of exotic atoms. The method uses a commercially available $\mathrm{x}$-ray diffraction spectrometer and a general-purpose laser. For bending radii of $3 \mathrm{~m}$, in the worst case an accuracy of $0.02^{\circ}$ was achieved for the cut angle, which is easily enhanced to a few millidegrees when using a better $y$ - $z$ table.

\section{ACKNOWLEDGMENTS}

The authors thank the Laboratory for Developments and Methods at PSI and, in particular, Dr. H. Grimmer, who made possible the use of the SEIFERT ${ }^{\circledR}$ spectrometer and gave his advice for operating the machine. We are grateful to A. Schmidheiny from the PSI HF group for providing the mechanical support. The Bragg crystals were manufactured by Carl Zeiss AG, Oberkochen, Germany. D.S.C. has a Ph.D. scholarship granted by FCT (Lisbon) through the POCI 2010 program.

${ }^{1}$ D. Gotta, Prog. Part. Nucl. Phys. 52, 133 (2004).

${ }^{2}$ D. F. Anagnostopoulos, S. Biri, D. Gotta, A. Gruber, P. Indelicato, B. Leoni, H. Fuhrmann, L. M. Simons, L. Stingelin, A. Wasser, and J. Zmeskal, Nucl. Instrum. Methods Phys. Res. A 545, 217 (2005).

${ }^{3}$ D. Gotta, F. Amaro, D. F. Anagnostopoulos, S. Biri, D. S. Covita, H. Gorke, A. Gruber, M. Hennebach, A. Hirtl, T. Ishiwatari, P. Indelicato, Th. Jensen, E.-O. Le Bigot, J. Marton, M. Nekipelov, J. M. F. dos Santos, S. Schlesser, Ph. Schmid, L. M. Simons, Th. Strauch, M. Trassinelli, J. F. C. A. Veloso, and J. Zmeskal, Lect. Notes Phys. 745, 165 (2008).

${ }^{4}$ H. H. Johann, Z. Phys. 69, 185 (1931).

${ }^{5}$ J. Eggs and K. Ulmer, Z. Angew. Phys. 5, 118 (1965).

${ }^{6}$ A. Guinier, Comptes rendus hebdomadaires des séances de I'l Académie des Sciences 223, 31 (1946).

${ }^{7}$ M. Sanchez del Rio and F. Cerrina, Rev. Sci. Instrum. 63, 936 (1992).

${ }^{8}$ M. Sanchez del Rio and R. J. Dejus, AIP Conf. Proc. 705, 784 (2004).

${ }^{9}$ G. Filscher, F. Adolph, and L. Adler, Krist. Tech. 9, 437 (1974).

${ }^{10}$ S. E. G. Slusky and A. T. Macrander, Appl. Phys. Lett. 53, 2042 (1988).

${ }^{11}$ B. K. Tanner, S. J. Miles, G. G. Peterson, and R. N. Sacks, Mater. Lett. 7, 239 (1988).

${ }^{12}$ Y. Chen, M. F. Morris, B. Obradovic, D. Li, Al. F. Tasch, and J. S. Swinnea, IEEE Trans. Semicond. Manuf. 13, 243 (2000).

${ }^{13}$ M. Gailhanou, Appl. Phys. Lett. 63, 458 (1993).

${ }^{14}$ L. D. Doucette, M. Pereira da Cunha, and R. J. Lad, Rev. Sci. Instrum. 76, 036106 (2005). 